\title{
The Gamma-ray Blazar Quest: new optical spectra, state of art and future perspectives
}

\author{
F. Massaro ${ }^{1,2}$, N. Álvarez Crespo ${ }^{1,2}$, R. D'Abrusco ${ }^{3}$, M. \\ Landoni $^{4}$, N. Masetti ${ }^{5,6}$, F. Ricci ${ }^{7}$, D. Milisavljevic ${ }^{8}$, A. \\ Paggi $^{8}$, V. Chavushyan ${ }^{9}$, E. Jiménez-Bailón ${ }^{10}$, V. \\ Patiño-Álvarez ${ }^{9}$, J. Strader ${ }^{11}$, L. Chomiuk ${ }^{11}$, F. La Franca ${ }^{7}$, \\ Howard A. Smith ${ }^{4}$ \& G. Tosti ${ }^{12}$
}

\begin{abstract}
We recently developed a procedure to recognize $\gamma$-ray blazar candidates within the positional uncertainty regions of the unidentified/unassociated $\gamma$-ray sources (UGSs). Such procedure was based on the discovery that Fermi blazars show peculiar infrared colors. However, to confirm the real nature of the selected candidates, optical spectroscopic data are necessary. Thus, we performed an extensive archival search for spectra available in the literature in parallel with an optical spectroscopic campaign aimed to reveal and confirm the nature of the selected $\gamma$-ray blazar candidates. Here, we first search for optical spectra of a
\end{abstract}

F. Massaro, N. Álvarez Crespo, R. D’Abrusco, M. Landoni, N. Masetti, F. Ricci, D. Milisavljevic, A. Paggi, V. Chavushyan, E. Jiménez-Bailón, V. Patiño-Álvarez, J. Strader, L. Chomiuk, F. La Franca, Howard A. Smith \& G. Tosti

${ }^{1}$ Dipartimento di Fisica, Università degli Studi di Torino, via Pietro Giuria 1, I-10125 Torino, Italy

${ }^{2}$ Istituto Nazionale di Fisica Nucleare, Sezione di Torino, I-10125 Torino, Italy

${ }^{3}$ Department of Physical Sciences, University of Napoli Federico II, via Cinthia 9, 80126 Napoli, Italy

${ }^{4}$ INAF-Osservatorio Astronomico di Brera, Via Emilio Bianchi 46, I-23807 Merate, Italy

${ }^{5}$ INAF - Istituto di Astrofisica Spaziale e Fisica Cosmica di Bologna, via Gobetti 101, 40129, Bologna, Italy

${ }^{6}$ Departamento de Ciencias Físicas, Universidad Andrés Bello, Fernández Concha 700, Las Condes, Santiago, Chile

${ }^{7}$ Dipartimento di Matematica e Fisica, Università Roma Tre, via della Vasca Navale 84, I-00146, Roma, Italy

${ }^{8}$ Harvard - Smithsonian Center for Astrophysics, 60 Garden Street, Cambridge, MA 02138, USA

${ }^{9}$ Instituto Nacional de Astrofísica, Óptica y Electrónica, Apartado Postal 51-216, 72000 Puebla, México

${ }^{10}$ Instituto de Astronomía, Universidad Nacional Autónoma de México, Apdo. Postal 877, Ensenada, 22800 Baja California, México

${ }^{11}$ Department of Physics and Astronomy, Michigan State University, East Lansing, MI 48824, USA

${ }^{12}$ Dipartimento di Fisica, Università degli Studi di Perugia, 06123 Perugia, Italy selected sample of $\gamma$-ray blazar candidates that can be potential counterparts of UGSs using the Sloan Digital Sky Survey (SDSS DR12). This search enables us to update the archival search carried out to date. We also describe the state-of-art and the future perspectives of our campaign to discover previously unknown $\gamma$-ray blazars.

Keywords galaxies: active - galaxies: BL Lacertae objects - quasars: general - surveys - radiation mechanisms: nonthermal

\section{Introduction}

The first observations performed with the Energetic Gamma Ray Experiment Telescope (EGRET; Thompson et al.1993) revealed that the largest fraction of sources detected in the $\mathrm{MeV}-\mathrm{GeV}$ energy range were unidentified/unassociated. According to the latest versions of the $3^{\text {rd }}$ EGRET (Hartman et al. 1999 Casandjian \& Grenier 2008) source catalog, the fraction of $\gamma$-ray objects with an unknown origin is $\sim 60 \%$. This is mostly due to the large positional uncertainties of the $\gamma$ ray sources being up to an order of magnitude greater than those at lower energies. Thus, the association of $\gamma$-ray sources with their low energy counterparts is one of the most demanding task for modern $\gamma$-ray astronomy (see e.g., Thompson 2008; Massaro et al. 2012a; Massaro et al. 2016).

The study of the unidentified/unassociated $\gamma$-ray sources (UGSs) was set as one of the major scientific goals of the Fermi mission (Atwood et al. 2009). Thanks to the major improvements achieved in the source localization with the Large Area Telescope (LAT) on board of Fermi, the "source association task" has been greatly simplified. This was also due to the improvements of statistical methods used to search for low energy counterparts of Fermi detected objects (see e.g., Abdo et al. 2010a; Abdo et al. 2010b). Difficulties on the source association in $\gamma$-rays is partially mitigated by another aspect: the largest fraction of known $\gamma$-ray 
emitters is associated with the rarest class of active galaxies: the blazars. In the Fermi-Large Area Telescope (LAT) third source catalog (3FGL; Acero et al. 2015) blazars constitute about $36 \%$ of the Fermi sources.

Blazars are generally divided in two classes on the basis of the equivalent width (EW) of their optical spectral features (see e.g., Stickel et al. 1991). When presenting featureless spectra or with emission/absorption lines with $\mathrm{EW}<5 \AA$ they are classified as BL Lac objects, while those showing quasar-like optical spectra coupled with a flat radio spectrum are known as flat spectrum radio quasars (FSRQ; see also Urry \& Padovani 1995, for more details on the blazar properties). Following the nomenclature proposed in the multifrequency catalog of Blazars (Roma-BZCAT; Massaro et al. 2015b), we indicate the former class as BZB while the latter one as BZQ. We also considered the blazar of galaxy type (indicated as BZG), according to the definition presented in the latest release of the Roma-BZCAT catalog ${ }^{1}$ (Massaro et al. 2009), being sources whose multifrequency emission exhibits some properties of blazars but appears dominated by the host galaxy contribution in the optical-ultraviolet energy range (Massaro et al. 2012b, Massaro et al. 2014).

Across all the releases of the Fermi-LAT source catalogs, the fraction of UGSs does not seem to decrease significantly. In the First Fermi-LAT source catalog (1FGL Abdo et al. 2010a) there were 630 out of 1451 unassociated Fermi objects (i.e., $\sim 43 \%$ ), fraction that decreases to $34 \%$ in the Second Fermi-LAT source catalog (2FGL Nolan et al. 2012) then being $~ 33 \%$ in the 3FGL. This is in agreement with the fact that even as more distant and/or less luminous sources are found, the UGS fraction remains about the same. This indicates that, even eight years after the launch of Fermi, unveiling the UGS nature is still an unsolved issue.

According to the 3FGL analysis the second largest population is constituted by the blazar candidates of uncertain type (BCUs) defined in the 3FGL and in the Third Catalog of Active Galactic Nuclei Detected by the FermiLAT (3LAC; Ackermann et al. 2015a see following sections for additional details). This corresponds to a revised definition of the previous $\gamma$-ray source class of active galaxies of uncertain type (AGUs) (see e.g., the First and the Second Catalog of Active Galactic Nuclei Detected by the Fermi-LAT, 1LAC and 2LAC, respectively Abdo et al. 2010b, Ackermann et al. 2011). BCUs are most probably all blazars (see e.g., Massaro et al. 2012c Álvarez-Crespo et al. 2016c, Chiaro et al. 2016), but the lack of spectroscopic information does not permit us to confirm their nature.

\footnotetext{
${ }^{1}$ http://www.asdc.asi.it/bzcat/
}

Follow up observations aiming to search for the potential counterparts of the UGSs and to confirm BCUs have been carried out in the radio (e.g., Kovalev 2009; Hovatta et al. 2012; Petrov et al. 2013; Hovatta et al. 2014; Schinzel et al. 2015), even below 1GHz (Massaro et al. 2013a; Nori et al. 2014; Giroletti et al. 2016), in the sub millimeter ranges (Giommi et al. 2012, López-Caniego et al. 2013) and in the X-rays with Swift (e.g., Mirabal \& Halpern 2009, Paggi et al. 2013; Takeuchi et al. 2013, Stroh et al. 2013; Acero et al. 2013) as well as with Chandra and Suzaku (e.g., Maeda et al. 2011, Cheung et al. 2012, Kataoka et al. 2012; Takahashi et al. 2012). Additional archival analyses were also carried out using multifrequency surveys and catalogs (see e.g., Cowperthwaite et al. 2013; Maselli et al. 2013; Maselli et al. 2015).

Using the WISE all-sky survey (Wright et al. 2010), we showed that in the IR color-color diagrams the $\gamma$-ray blazars, dominated by non-thermal emission, lie in a distinct region well separated from that occupied by other extragalactic sources (Massaro et al. 2011a, D'Abrusco et al. 2012; Massaro \& D'Abrusco 2016). On the basis of this discovery, we built new procedures to recognize $\gamma$-ray blazar candidates lying within the positional uncertainty regions of the UGSs (D'Abrusco et al. 2013; Massaro et al. 2013b; D'Abrusco et al. 2014).

Then in 2012 we started an optical spectroscopic campaign to confirm the nature of both $\gamma$-ray blazar candidates selected according to our IR procedure, UGS potential counterparts and BCUs associated in the Fermi catalogs. All the spectroscopic observations collected during our campaign are already published (see e.g., Paggi et al. 2014; Massaro et al. 2015c; Landoni et al. 2015; Ricci et al. 2015 Álvarez-Crespo et al. 2016a; Álvarez-Crespo et al. 2016b). During our campaign we also continuously searched in the optical databases to exclude targets for which an optical spectrum became recently available (see e.g., Massaro et al. 2014, Massaro et al. 2015a, Álvarez Crespo et al. 2016c, for the spectra of the Sloan Digital Sky Survey, SDSS, DR12 and of the Six-degree-Field Galaxy Survey, 6dFGS).

In the present paper we aim to present the state-of-art of our hunt for $\gamma$-ray blazar candidates. Scientific objectives of the current analysis can be summarized as follows:

1. searching for optical spectra of radio sources that could be potential counterparts of 3FGL UGSs lying in the SDSS footprint;

2. presenting an overview of the results of our optical spectroscopic campaign achieved to date;

3. discussing on the future perspectives towards the preparation of the 4FGL catalog.

The paper is organized as follows. In $\S 2$ we described our search for radio sources having a blazar-like optical spectrum and lying within the positional uncertainty region of the UGSs in the footprint of the SDSS. Then $\S 3$ and $\S 4$ 
are devoted to present results and the summary of our optical spectroscopic campaign, respectively. Finally in $\S 5$ we speculate on future perspectives towards a better understanding of the unknown $\gamma$-ray sky.

We used cgs units unless stated otherwise through the whole paper, and spectral indices, $\alpha$, are defined by flux density, $\mathrm{S}_{v} \propto v^{-\alpha}$ considering sources with a flat spectrum when $\alpha<0.5$.

\section{Searching for $\gamma$-ray BL Lac candidates in the footprint of the Sloan Digital Sky Survey}

SDSS J214530.19+100605.4 (3FGL J2145.5+1007)

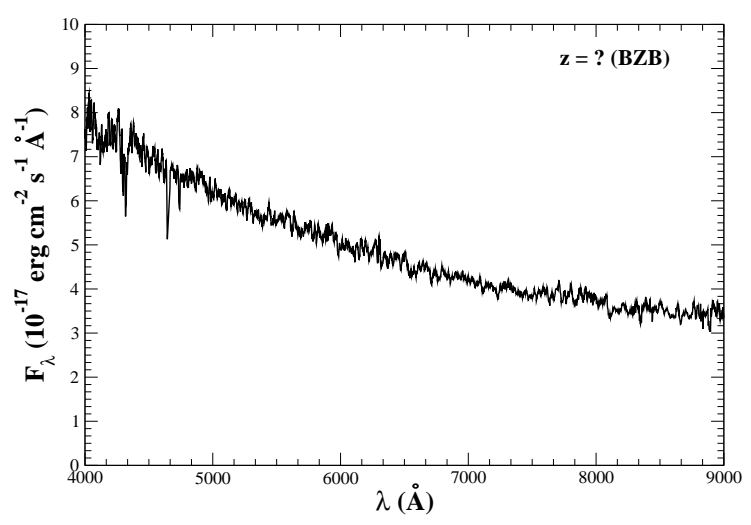

Fig. 1 The optical spectrum of SDSS J214530.19+100605.4, classified as a BZB at unknown redshift, lying within the positional uncertainty region of the UGS 3FGL J2145.5+1007.

As previously stated, our optical spectroscopic campaign was carried out both observing $\gamma$-ray blazar candidates and searching major optical databases and surveys. To perform the literature search we crossmatched the radio/IR position of the $\gamma$-ray blazar candidates selected for the UGSs as well as those of the BCUs reported in the Fermi catalogs with those reported in the comparison survey as, for example, the SDSS. Thus to complete and update our literature search we decided to carry out the following additional analysis.

We considered all the UGSs lying in the footprint of the SDSS DR12 (Alam et al. 2015). Then we searched for all sources having the SDSS spectrum available and with a radio counterpart in the Faint Images of the Radio Sky at Twenty-centimeters (FIRST) survey (see e.g., Becker et al. 1995: White et al. 1997). Finally, we selected only those SDSS sources with a BL Lac or a quasar-like optical spectrum. The latter are considered blazar-like when showing a flat radio spectrum.

This further investigation led to the discovery of six new BL Lacs and one BZQs, potential counterparts of Fermi
UGSs, since one BZQ and two BZBs were recently reported in the latest release of the Roma-BZCAT but not in the 3FGL. The spectra of one BL Lac is shown in Fig 1 as template of those selected in our analysis. Source details for these eleven blazars are reported in Table 1 where we report (i) their 3FGL names together with those of the SDSS and the WISE potential low energy counterparts; (ii) our classification; (iii) the redshift estimate when possible; (iv) alternative Fermi names from previous catalogs and (v) the RomaBZCAT name.

It is worth noting that the UGS 3FGL J0158.6+0102 could be potentially associated with SDSS J015852.76+010132.9 classified as BZU in the latest release of the Roma-BZCAT 5BZU J0158+0101 but appearing as a BL Lac object in the SDSS presented here (see Fig. 2). Then we also remark that 3FGL J2223.3+0103 is missing from 3LAC but present in 2FGL class AGU without redshift.

Finally, we emphasize that even if four blazars are already present in the latest version of the Roma-BZCAT (Massaro et al. 2015c) there were not indicated as counterparts of 3FGL sources because the latter catalog was released previously.

\section{State-of-art of our optical spectroscopic campaign}

Here we present the summary of the results achieved during spectroscopic campaign considering the latest release of the Fermi catalog available to date (i.e., 3FGL). Given the differences in the $\gamma$-ray analysis between the releases of the Fermi-LAT source catalogs we also discuss some targets that belong to the 1FGL and the 2FGL catalogs only.

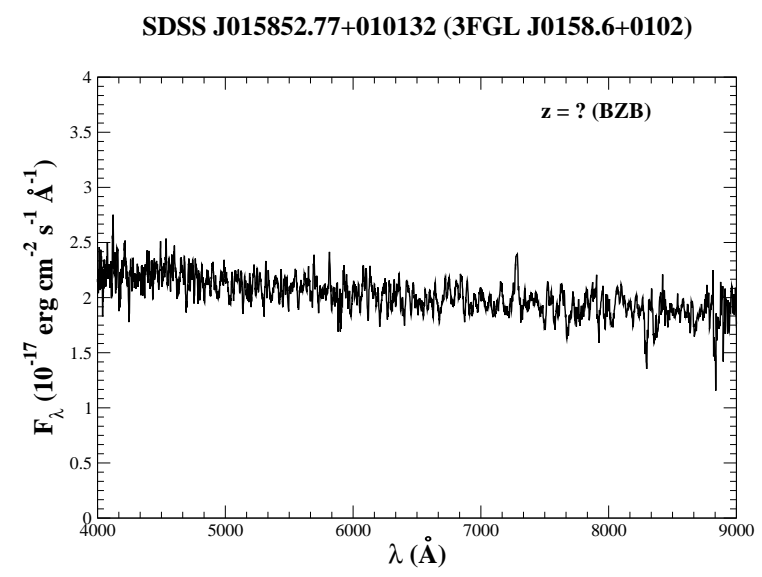

Fig. 2 The featureless optical spectrum of SDSS J015852.76+010132.9, potential low energy counterpart of the unassociated Fermi source: 3FGL J0158.6+0102. The source is classified by us as a BL Lac object. 
Table 1 Summary of source details for the eleven blazars found within the sample of UGSs lying in the SDSS footprint (See $\S 2$ for more details).

\begin{tabular}{|c|c|c|c|c|c|c|c|c|}
\hline $\begin{array}{l}\text { 3FGL } \\
\text { name }\end{array}$ & $\begin{array}{l}\text { SDSS } \\
\text { name }\end{array}$ & $\begin{array}{l}\text { WISE } \\
\text { name }\end{array}$ & class & $z$ & $\begin{array}{l}\text { 1FGL } \\
\text { name }\end{array}$ & $\begin{array}{l}\text { 2FGL } \\
\text { name }\end{array}$ & $\begin{array}{l}\text { 1FHL } \\
\text { name }\end{array}$ & $\begin{array}{l}\text { BZCAT } \\
\text { name }\end{array}$ \\
\hline J0158.6+0102 & J015852.77+010132.9 & J015852.76+010132.9 & bzb & 0.0 & & J0158.4+0107 & & 5BZU J0158+0101 \\
\hline J0234.2-0629 & J023410.30-062825.7 & J023410.28-062825.8 & bzb & 0.0 & & & & \\
\hline J1103.3+5239 & J110249.84+525012.6 & J110249.86+525012.6 & bzq & 0.68984 & & & & \\
\hline$J 1105.7+4427$ & J110544.28+442830.4 & J110544.29+442830.6 & bzb & 0.74641 & & & & \\
\hline J1129.0+3758 & J112904.78+375844.6 & J112904.78+375844.6 & bzb & 0.0 & J1129.3+3757 & J1129.5+3758 & & \\
\hline $\mathrm{J} 1301.5+3333$ & $\mathrm{~J} 130129.14+333700.3$ & $\mathrm{~J} 130129.16+333700.2$ & bzq & 1.00826 & & $\mathrm{~J} 1301.6+3331$ & & 5BZQ J1301+3337 \\
\hline J1330.4+5641 & $\mathrm{J} 133040.69+565520.1$ & $\mathrm{~J} 133040.67+565520.1$ & bzb & 0.0 & & & & $5 \mathrm{BZB}$ J1330+5655 \\
\hline $\mathrm{J} 1411.1+3717$ & $\mathrm{~J} 141130.56+372245.5$ & $\mathrm{~J} 141130.51+372246.4$ & bzb & 0.0 & & & & \\
\hline $\mathrm{J} 1731.9+5428$ & $\mathrm{~J} 173340.32+542636.8$ & $\mathrm{~J} 173340.31+542636.7$ & bzb & 0.0 & & $\mathrm{~J} 1730.8+5427$ & & \\
\hline J2145.5+1007 & $\mathrm{J} 214530.19+100605.4$ & $\mathrm{~J} 214530.19+100605.5$ & bzb & 0.0 & & & & \\
\hline $\mathrm{J} 2223.3+0103$ & J222329.57+010226.6 & $\mathrm{J} 222329.57+010226.7$ & bzb & 0.0 & $\mathrm{~J} 2223.3+0103$ & J2223.4+0104 & J2223.4+0104 & 5BZB J2223+0102 \\
\hline
\end{tabular}

We only observed those AGUs that had not been otherwise observed at the time of the 2FGL release (see e.g., Shaw et al. 2013a; Shaw et al. 2013b). On the other hand, we recently provided an important contribution to the classification of the 3FGL BCUs obtaining optical spectra for about 1/5 of them (see e.g., Massaro et al. 2015a, and references therein).

Regarding the UGSs the impact of our campaign was crucial to decrease their percentage in the 1FGL and in the 2FGL (see also Massaro et al. 2015a, for a recent review on the sources listed in both these Fermi catalogs), while for the 3FGL the number of newly discovered blazars correspondent to the UGSs is rather small (see following Section). This is mostly due to the fact that the catalog of IR selected blazar-like sources (D'Abrusco et al. 2014) was used to search for associations of the 3FGL sources, thus providing a significant number of BCUs that we preferred to follow up during our spectroscopic campaign.

\subsection{General Results}

All the 3FGL sources observed to date are reported in Table 2 , 3 and 4 where we provide (i) their Fermi names together with their (ii) $\gamma$-ray classification, when present; (iii) the associated counterpart for the BCUs and (iv) the infrared (i.e., WISE or 2MASS) name of the counterpart observed in our campaign; then we also present (v) our optical classification: BZB for BL Lac objects, BZQ for flat spectrum radio quasars and quasar (QSO) for sources having no radio counterpart and/or not a flat radio spectrum but a quasar-like (i.e., broad emission lines) optical behavior, respectively. Then we also show (vi) the reference among our campaign papers, (vii) the Roma-BZCAT name. For our optical classification we adopted the Roma-BZCAT criteria, including the definition of blazar of galaxy type (BZG) for sources having an SED with a significant dominance of the host galaxy emission over the nuclear one (see Massaro et al. 2015b, for more details). It is also worth noting that QSOs, having no blazar-like radio emission, could be contaminants of our selection procedure, as described in D'Abrusco et al. (2014).
The $\gamma$-ray classification reported in Table ??, 3 and 4 corresponds to the one of the 3LAC (Ackermann et al. 2015a). In particular we distinguish among BCU of type I, II and III on the basis of the following criteria. These corresponds to the $a, b, c$ subclasses of the $3 \mathrm{LAC}$, respectively.

(I) a BZU object (blazar of uncertain/transitional type) in the Roma-BZCAT;

(II) a source with multiwavelength data in one of the catalogs used for the 3LAC associations, with a flat radio spectrum, and a typical two-humped, blazar-like spectral energy distribution (SED);

(III) a source with a radio and an X-ray counterparts also showing a typical two-humped, blazar-like SED.

Since the beginning of our follow up spectroscopic observations in 2012 we pointed 198 unique targets correspondent to 3 FGL sources.

For 3FGL sources we summarize our results as reported below.

- We found 75 BZBs all classified in the 3FGL as BL Lacs with the only exception of that associated with 3FGL J2356.0+4037 appeared as a BZG. Most of these targets (i.e., more than $\sim 60 \%$ ) were unclassified counterparts of 2FGL sources for which their nature is actually reported in the 3FGL thanks to our campaign. This occurred because a significant fraction of our observations begun between the 2FGL and the 3FGL releases.

- Nine 3FGL sources associated with flat spectrum radio quasars were also observed, all resulting as BZQ and with a $z$ estimate.

- We observed 88 BCUs. In this sample we found : 14 BZQs and 10 BZGs all with known redshifts; 1 quasar and 1 source with still uncertain classification plus 62 BZBs, 6 also having a good redshift estimate and 2 with an uncertain $z$ value.

- We observed 26 blazar-like objects that could be potential counterparts of Fermi UGSs, selected with their IR colors. For six UGSs we pointed a quasar, most probably a contaminant of our selection methods unless future radio follow up observations will reveal a flat radio spectrum. The remaining sources are indeed 
all blazar-like. We found 2 BZQs, one with known redshift, 1 BZG with a certain $z$ estimate and 17 BZBs with a certain $z$ value reported in only four cases.

We also observed 10 unique targets that belong to 1FGL, 2FGL and the First Fermi-LAT Catalog of Sources above $10 \mathrm{GeV}$ (1FHL; Ackermann et al. 2013) catalogs but are not in the 3FGL. Results on this sample are thus summarized below.

- The potential counterparts of 1FHL J0030.1-1647 and 1FHL J0044.0-1111, both UGS of the 1FHL catalog, could be potentially associated to two newly discovered BZBs at known redshifts, 0.237 and 0.264 , respectively (Álvarez-Crespo et al. 2016b).

- For the three 1FGL sources we found: 1FGL J0411.6+5459 potentially associated to a new BL Lac at unknown $z$; one QSO contaminant of our selection method for the UGS 1FGL J2110.3+3820; and 1FGL J1422.7+3743 for which we confirmed the BZB classification available in the literature.

- We observed 3 AGUs of the 2FGL subsample all classified as BZQs and all with a certain redshift estimate provided. Then we also confirm that 2FGL J0819.60803 is associated with a BZB at redshift unknown. One additional UGS, 2FGL J1745.6+0203, was also observed searching for blazar-like sources within the Fermi positional uncertainty region, but we pointed another QSO.

\subsection{Additional source details}

During our campaign we also re-observed a few sources, mostly BL Lacs for which the redshift $z$ estimate was uncertain or unknown, to obtain a better measurement and also to catch some transitional states as occurred in a couple of cases (see Álvarez Crespo et al. 2016a,b). In three cases we also pointed a different target than that actually reported in the 3LAC as described below. Thus we also add the following notes with respect to the results presented in the published papers and previously summarized.

- In Álvarez Crespo et al. (2016a) for the source 3FGL J0653.6+2817 associated with the BCU: GB6 J0653+2816 we pointed a different target resulting as a BZG: WISE J065340.46+281848.5 at uncertain $z>0.45$ instead of WISE J065344.26+281547.5. The same occurred for the BCU associated with 3FGL J1013.5+3440 for which WISE J101336.51+344003.6 was observed instead of OL 318 . In addition to them, in Massaro et al. (2015a) for 3FGL J1549.0+6309 the reported "candidate association" pointed during our campaign is: NVSS J154828+631050. The source associated in the 3FGL/3LAC catalog has the coordinates of SDSS J154957.32+631007.3 (a.k.a. CRATES $\mathrm{J} 1549+6310$ ) but the counterpart name seems incorrect being: SDSS J154958.45+631021.2; in both cases out pointed target differs from them.
- The BCU NVSS J132210+084231 associated with 3FGL J1322.3+0839 was observed in Álvarez Crespo et al. (2016a) and Álvarez Crespo et al. (2016c) in a different state, since it appeared as a BZB first and a BZQ at $z=0.32549$ later. The optical spectrum of 3FGL J0158.6+0102 is reported Massaro et al. (2014) as well as in the current paper. This source showed a BZQ spectrum in the past not confirmed by the most recent observation. Thus these two sources can be considered as transitional sources present in our sample in addition to those discovered in (Álvarez Crespo et al. 2016a,c): 3FGL J0433.1+3228 and 3FGL J1040.4+0615. These two Fermi objects were originally classified as BZQ while had a typical BZB optical spectrum when pointed by us.

- Only for the AGU: TXS 1801+253 associated in the 2FGL with 2FGL J1803.6+2523c, we were not able to find a WISE counterpart, due to a confusion with a nearby star (see Álvarez-Crespo et al. 2016c for more details).

- We re-pointed the associated counterparts of 3FGL J1340.6-0408, 3FGL J1314.8+2349, 3FGL J2323.9+4211, 3FGL J1129.0+3758 and 3FGL J2223.3+0103 during our campaign but they always appeared as BZBs at unknown redshift.

- We found that the associated counterparts of 3FGL J0522.9-3628 is a BZQ while those of 3FGL J0009.63211 and 3FGL $\mathrm{J} 2356.0+4037$ are indeed BZGs at redshifts 0.002542 and 0.131 , respectively.

\section{Summary and Conclusions}

The WISE all-sky survey (Wright et al. 2010) allowed us to distinguish $\gamma$-ray emitting blazars from other extragalactic sources. On the basis of the mid-IR colors we developed a set of procedures to select potential counterparts of the UGSs and to recognize blazar candidates within the AGUs and the BCUs listed in the Fermi catalogs. Motivated by preliminary results of our IR selection, we carried out an optical spectroscopic campaign aiming to verify the blazar-like nature of UGS potential counterparts As secondary goal we also obtained a classification for AGUs and BCUs present in the Fermi catalogs.

During our campaign we collected and analyzed 223 unique spectra and the major results achieved are summarized as follows.

1. The largest fraction of pointed targets are classified as BZBs (i.e., 173/223). This strongly suggests that Fermi survey is extremely useful to discover new BZBs and confirms that these are one of most elusive class of active galaxies. 
6

2. We obtained 49 certain redshifts for the blazars observed thanks to the presence of emission/absorption lines or only absorption features due to intervening systems.

3. We discovered a handful of transitional sources: blazars with a different spectrum available in the literature and thus classified differently.

4. During our campaign we found two BL Lac objects without radio counterparts in the major radio surveys (Paggi et al. 2014; Ricci et al. 2015). This discovery could open new scenarios on the blazar phenomenon and could potentially make the $\gamma$-ray association task more challenging since a large fraction of the Fermi associations come from radio surveys/catalogs.

5. Within the sample of newly discovered BL Lacs with a $z$ estimate, presented in Section 2, it is worth noting that one of them lie above redshift $z=0.7$ (this occurs only for $11 \mathrm{BL}$ Lacs from the Roma-BZCAT listed in the Fermi catalogs).

Given the small number of QSOs found during our campaign (i.e., $\sim 4 \%$ ) in addition to a handful of unpublished spectra that did not have enough signal-to-noise ratio to clearly classify the observed targets, our results strongly supports the reliability of our IR selection method (see D'Abrusco et al. 2016, for more details).

Finally, we emphasize that results obtained thanks to our campaign were used to classify the sources listed in the latest release of the Fermi catalogs (i.e., 3FGL, 3LAC and 2FHL Ackermann et al. 2016) as well as to increase those in the Roma-BZCAT.

\section{Future perspectives}

Our spectroscopic follow up campaign at optical wavelengths is still on-going and we expect to have an additional $\sim 80$ targets presented by the end of 2016 .

These optical observations of $\gamma$-ray blazar candidates are crucial to build the luminosity function of BL Lacs (see e.g., Ajello et al. 2012, Ajello et al. 2014), confirm potential targets for the Cherenkov Telescope Array (CTA) selected on the basis of multifrequency properties (see e.g., Massaro et al. 2013c, Arsioli et al. 2015), obtain stringent limits on the dark matter annihilation in sub-halos (see e.g., Zechlin \& Horns 2012; Berlin \& Hooper 2014) and/or from the estimates of the extragalactic $\gamma$-ray background (EGB Ajello et al. 2015), search for counterparts of new flaring $\gamma$-ray sources (see e.g. Bernieri et al. 2013) and identify potential counterparts of new Fermi sources discovered with detection algorithms different from those used to build the Fermi catalog (see e.g., Campana et al. 2015. Campana et al. 2016a; Campana et al. 2016b). Studies on the UGSs are also crucial to discover new BL Lacs that might improve our knowledge on their contribution to the extragalactic $\gamma$-ray background (EGB; see e.g., Ajello et al. 2014).

The BL Lac contribution considered together with that arising from other $\gamma$-ray emitters as for example radiogalaxies (see e.g. Inoue 2011, Massaro \& Ajello 2011b; Di Mauro et al. 2014) and/or star forming galaxies (see e.g., Ackermann et al. 2012a) could help searching for a potential dark matter signature in the EGB spectrum (Ackermann et al. 2015a, Di Mauro \& Donato 2015, Fermi Collaboration 2015). Moreover optical spectroscopic campaign are also useful to obtain new redshifts necessary to estimate the imprint of the extragalactic background light in their spectra (see e.g., Ackermann et al. 2012b).

At the current moment the major problem we foresee to continue after 2016 resides in the strategy originally developed, even if it has been successful to date. To minimize the impact on telescope schedules and maximize the scientific return, we proposed a small subsample of targets each time. Consequently, each publication presents only a limited amount of spectra, in the range between 20 and 50 . However, it is worth noting that even if the number of the Fermi sources for each catalog is growing consistently with the expectations: 1451 in the 1FGL, 1873 in the 2FGL and now 3033 in the 3FGL, the fraction of UGSs is rather constant. Thus if the expected number of sources in the next Fermi catalog (i.e., 4FGL) will be $\sim 4500$ sources, the number of UGSs and BCUs expected of the order of 2000. Consequently, assuming we can still obtain observing nights at the telescopes used to date, collecting 200 spectra in about 3 years, it will be impossible to achieve our final goal of "resolving" the $\gamma$-ray sky on a few years time scale.

To complete our campaign, considering the sources listed in the 3FGL, the number of targets that must be observed is approximately 550, mostly concentrated in the Southern hemisphere, and correspondent to $\sim 40$ observing nights, assuming to use 4 meter class telescopes/facilities. Thus, the only strategy suitable to achieve our final goal is a survey/large program. Completing these observations will allow us to assign a certain classification to all the BCUs listed in the 3FGL and decrease the fraction of 3FGL UGSs by a factor of $5-10 \%$, assuming results similar to those achieved to date.

We thank the anonymous referee for useful comments that led to improvements in the paper. F.M. gratefully acknowledges the financial support of the Programma Giovani Ricercatori - Rita Levi Montalcini - Rientro dei Cervelli (2012) awarded by the Italian Ministry of Education, Universities and Research (MIUR). This investigation is supported by the NASA grants NNX12AO97G and NNX13AP20G. The work by G. Tosti is supported by the ASI/INAF contract I/005/12/0. H. A. Smith acknowledges partial support from NASA/JPL grant NNX14AJ61G. H. 
Otí-Floranes is funded by a postdoctoral UNAM grant. V. Chavushyan acknowledges funding by CONACyT research grant 151494 (México). We thank the staff at the Observatorio Astronómico Nacional in San Pedro Mártir (México) for all their help during the observation runs. We thank the staff of the Astronomical Observatory of Bologna in Loiano for their assistance during the observations. Part of this work is based on archival data, software or on-line services provided by the ASI Science Data Center. This research has made use of data obtained from the high-energy Astrophysics Science Archive Research Center (HEASARC) provided by NASA's Goddard Space Flight Center; the SIMBAD database operated at CDS, Strasbourg, France; the NASA/IPAC Extragalactic Database (NED) operated by the Jet Propulsion Laboratory, California Institute of Technology, under contract with the National Aeronautics and Space Administration. This publication makes use of data products from the Wide-field Infrared Survey Explorer, which is a joint project of the University of California, Los Angeles, and the Jet Propulsion Laboratory/California Institute of Technology, funded by the National Aeronautics and Space Administration. This publication makes use of data products from the Two Micron All Sky Survey, which is a joint project of the University of Massachusetts and the Infrared Processing and Analysis Center/California Institute of Technology, funded by the National Aeronautics and Space Administration and the National Science Foundation. Funding for SDSS-III has been provided by the Alfred P. Sloan Foundation, the Participating Institutions, the National Science Foundation, and the U.S. Department of Energy Office of Science. The SDSSIII web site is http://www.sdss3.org/. SDSS-III is managed by the Astrophysical Research Consortium for the Participating Institutions of the SDSS-III Collaboration including the University of Arizona, the Brazilian Participation Group, Brookhaven National Laboratory, Carnegie Mellon University, University of Florida, the French Participation Group, the German Participation Group, Harvard University, the Instituto de Astrofisica de Canarias, the Michigan State/Notre Dame/JINA Participation Group, Johns Hopkins University, Lawrence Berkeley National Laboratory, Max Planck Institute for Astrophysics, Max Planck Institute for Extraterrestrial Physics, New Mexico State University, New York University, Ohio State University, Pennsylvania State University, University of Portsmouth, Princeton University, the Spanish Participation Group, University of Tokyo, University of Utah, Vanderbilt University, University of Virginia, University of Washington, and Yale University. This research has made use of the USNOFS Image and Catalogue Archive operated by the United States Naval Observatory, Flagstaff Station (http://www.nofs.navy.mil/data/fchpix/). TOPCAT $^{2}$ (Taylor 2005) for the preparation and manipulation of the tabular data and the images. The Aladin Java

\footnotetext{
${ }^{2}$ http://www.star.bris.ac.uk/ mbt/topcat/
}

applet ${ }^{3}$ was used to create the finding charts reported in this paper (Bonnarell et al. 2000). It can be started from the CDS (Strasbourg - France), from the CFA (Harvard - USA), from the ADAC (Tokyo - Japan), from the IUCAA (Pune - India), from the UKADC (Cambridge - UK), or from the CADC (Victoria - Canada). 
Table 2 Summary of the targets observed during our optical spectroscopic campaign for the 3FGL sources. (R.A. 00-08)

\begin{tabular}{|c|c|c|c|c|c|c|c|}
\hline $\begin{array}{l}\text { 3FGL } \\
\text { name }\end{array}$ & $\begin{array}{l}\text { 3FGL } \\
\text { class }\end{array}$ & $\begin{array}{l}\text { 3FGL } \\
\text { counterpart }\end{array}$ & $\begin{array}{l}\text { counterpart } \\
\text { name }\end{array}$ & class & $z$ & reference & $\begin{array}{l}\text { Roma-BZCAT } \\
\text { name }\end{array}$ \\
\hline J0003.8-1151 & bcu II & PMN J0004-1148 & J000404.91-114858.3 & bzb & $\overline{0.0}$ & Crespo+16c & 5BZB J0004-1148 \\
\hline J0009.6-3211 & bcu I & IC 1531 & J000935.55-321636.8 & bzg & 0.02542 & Crespo+16c & \\
\hline J0015.7+5552 & bcu II & GB6 J0015+5551 & J001540.13+555144.7 & bzb & 0.0 & Crespo+16a & \\
\hline J0028.8+1951 & bcu III & TXS $0025+197$ & J002829.81+200026.7 & bzq & 1.5517 & Crespo+16c & \\
\hline J0030.2-1646 & bcu II & 1RXS J003019.6-164723 & J003019.40-164711.7 & bzb & 0.0 & Crespo+16c & \\
\hline J0043.5-0444 & bcu II & 1RXS J004333.7-044257 & J004334.12-044300.6 & bzb & 0.0 & Crespo $+16 c$ & \\
\hline J0103.4+5336 & bll & 1RXS J010325.9+533721 & J010325.89+533713.4 & bzb & 0.0 & Crespo+16a & \\
\hline J0103.7+1323 & bcu III & NVSS J010345+132346 & J010345.74+132345.3 & bzb & 0.49 & Crespo+16b & \\
\hline J0105.3+3928 & bll & GB6 J0105+3928 & J010509.19+392815.1 & bzb & 0.44 & Crespo+16b & 5BZB J0105+3928 \\
\hline J0109.1+1816 & bll & MG1 J010908+1816 & J010908.17+181607.5 & bzb & 0.0 & Massaro+14 & 5BZB J0109+1816 \\
\hline J0116.3-6153 & bll & SUMSS J011619-615343 & J011619.62-615343.4 & bzb & 0.0 & Landoni+15 & 5BZB J0116-6153 \\
\hline J0133.0-4413 & bll & SUMSS J013306-441422 & J013306.37-441421.4 & bzb & 0.0 & Landoni+15 & 5BZB J0133-4414 \\
\hline J0143.7-5845 & bll & SUMSS J014347-584550 & J014347.41-584551.4 & bzb & 0.0 & Landoni+15 & 5BZB J0143-5845 \\
\hline J0147.0-5204 & bcu I & PKS 0144-522 & J014648.58-520233.5 & bzg & 0.098 & Crespo $+16 c$ & 5BZG J0146-5202 \\
\hline J0148.3+5200 & bcu III & GB6 J0148+5202 & J014820.33+520204.9 & bzb & 0.0 & Crespo+16a & \\
\hline J0145.6+8600 & bcu III & NVSS J014929+860114 & J014935.28+860115.4 & bzg & 0.15 & Crespo+16a & \\
\hline J0156.9-4742 & bcu II & 2MASS J01564603-4744174 & J015646.03-474417.3 & bzb & 0.0 & Crespo+16c & \\
\hline J0157.0-5301 & bll & 1RXS J015658.6-530208 & J015658.00-530200.0 & bzb & 0.0 & Landoni +15 & 5BZB J0156-5302 \\
\hline J0158.6-3931 & bll & PMN J0158-3932 & J015838.10-393203.8 & bzb & 0.0 & Landoni+15 & 5BZB J0158-3932 \\
\hline J0158.6+0102 & & & J015852.76+010132.9 & bzb & 0.0 & Massaro+16 & 5BZU J0158+0101 \\
\hline J0158.6+0102 & & & J015852.76+010132.9 & bzq & $1.61 ?$ & Massaro+14 & 5BZU J0158+0101 \\
\hline J0219.0+2440 & bcu II & $87 \mathrm{~GB} 021610.9+243205$ & J021900.40+244520.6 & bzb & 0.0 & Crespo+16a & \\
\hline J0221.2+2518 & & & J022051.24+250927.6 & qso & 0.4818 & Paggi+14 & 5BZU J0220+2509 \\
\hline $\mathrm{J} 0222.6+4301$ & bll & $3 \mathrm{C} 66 \mathrm{~A}$ & $\mathrm{~J} 022239.60+430207.8$ & bzb & 0.0 & Paggi+14 & 5BZB J0222+4302 \\
\hline J0234.2-0629 & & & J023410.28-062825.8 & bzb & 0.0 & Massaro+16 & \\
\hline J0237.5-3603 & bll & RBS 0334 & J023734.04-360328.4 & bzb & 0.0 & Landoni+15 & 5BZB J0237-3603 \\
\hline J0238.4-3117 & bll & 1RXS J023832.6-311658 & J023832.48-311658.0 & bzb & 0.232 & Landoni+15 & 5BZB J0238-3116 \\
\hline J0253.5+3216 & bcu II & MG3 J025334+3217 & J025333.64+321720.8 & bzq & 0.859 & Ricci+15 & \\
\hline J0255.8+0532 & bcu II & PMN J0255+0533 & J025549.51+053355.0 & bzb & 0.0 & Crespo+16c & \\
\hline J0301.8-7157 & bcu II & PKS 0301-721 & J030138.47-715634.5 & bzq & 0.8232 & Crespo $+16 c$ & 5BZQ J0301-7156 \\
\hline J0307.3+4916 & & & J030727.21+491510.6 & bzb & 0.0 & Crespo+16b & \\
\hline J0309.5-0749 & bll & NVSS J030943-074427 & J030943.23-074427.5 & bzb & 0.0 & Massaro+15a & 5BZB J0309-0744 \\
\hline J0316.2-6436 & bll & SUMSS J031614-643732 & J031614.34-643731.4 & bzb & 0.0 & Landoni+15 & 5BZB J0316-6437 \\
\hline J0323.6-0109 & bll & 1RXS J032342.6-011131 & J032343.62-011146.1 & bzb & 0.0 & Massaro+14 & 5BZB J0323-0111 \\
\hline J0333.6+2916 & bll & TXS $0330+291$ & J033349.00+291631.5 & bzb & 0.0 & Crespo+16a & 5BZB J0333+2916 \\
\hline J0334.3-4008 & bll & PKS 0332-403 & J033413.65-400825.4 & bzb & 0.0 & Landoni+15 & 5BZB J0334-4008 \\
\hline J0335.3-4459 & bll & 1RXS J033514.5-445929 & J033513.88-445943.8 & bzb & 0.0 & Landoni+15 & 5BZB J0335-4459 \\
\hline J0339.2-1738 & bcu I & PKS 0336-177 & J033913.70-173600.8 & bzg & 0.06556 & Crespo+16c & \\
\hline $\mathrm{J} 0343.3+3622$ & bcu I & OE 367 & J034328.94+362212.4 & bzq & 1.485 & Crespo+16c & 5BZQ J0343+3622 \\
\hline J0352.9+5655 & bcu II & GB6 J0353+5654 & J035309.54+565430.7 & bzb & 0.0 & Crespo+16b & \\
\hline J0409.8-0358 & bll & NVSS J040946-040003 & J040946.58-040003.5 & bzb & 0.0 & Massaro+15a & 5BZB J0409-0400 \\
\hline J0415.7-4351 & & & J041605.82-435514.6 & qso & 0.398 & Landoni+15 & 5BZU J0416-4355 \\
\hline J0425.0-5331 & bll & PMN J0425-5331 & J042504.27-533158.2 & bzb & 0.0 & Landoni+15 & 5BZB J0425-5331 \\
\hline J0428.6-3756 & bll & PKS 0426-380 & J042840.41-375619.3 & bzb & $1.02 ?$ & Landoni+15 & 5BZB J0428-3756 \\
\hline J0433.1+3228 & bcu II & NVSS J043307+322840 & J043307.54+322840.7 & bzb & 0.0 & Crespo+16a & \\
\hline J0433.7-6028 & bcu II & PKS 0432-606 & J043334.59-603010.3 & bzq & 0.9301 & Crespo $+16 c$ & 5BZQ J0433-6030 \\
\hline J0434.4-2341 & bcu I & PMN J0434-2342 & J043428.98-234205.3 & bzb & 0.0 & Crespo+16c & 5BZB J0434-2342 \\
\hline J0439.9-1859 & bcu II & PMN J0439-1900 & J043949.72-190101.5 & bzb & 0.0 & Crespo+16c & \\
\hline J0505.9+6114 & bll & NVSS J050558+611336 & J050558.78+611335.9 & bzb & 0.0 & Paggi+14 & 5BZB J0505+6113 \\
\hline J0508.2-1936 & bcu II & PMN J0508-1936 & J050818.99-193555.7 & bzq & 1.88 & Crespo+16b & \\
\hline J0521.7+0103 & bcu II & NVSS J052140+010257 & J052140.82+010255.5 & ? & 0.0 & Crespo+16c & \\
\hline J0522.9-3628 & bcu I & PKS 0521-36 & J052257.98-362730.8 & bzq & 0.05655 & Crespo+16c & 5BZU J0522-3627 \\
\hline J0537.4-5717 & bll & SUMSS J053748-571828 & J053748.96-571830.1 & bzb & $1.18 ?$ & Landoni+15 & 5BZB J0537-5718 \\
\hline J0556.0-4353 & bll & SUMSS J055618-435146 & J055618.74-435146.0 & bzb & 0.0 & Landoni+15 & 5BZB J0556-4351 \\
\hline J0601.0+3837 & bll & PKS 0601-70 & J060102.86+383829.2 & bzb & 0.0 & Paggi+14 & 5BZB J0601+3838 \\
\hline J0604.1-4817 & bll & 1ES $0602-482$ & J060408.61-481725.1 & bzb & 0.0 & Landoni +15 & 5BZB J0604-4817 \\
\hline J0607.4+4739 & bll & TXS $0603+476$ & J060723.25+473947.0 & bzb & 0.0 & Massaro+15a & 5BZB J0607+4739 \\
\hline J0609.4-0248 & bll & NVSS J060915-024754 & J060915.06-024754.5 & bzb & 0.0 & Massaro+15a & 5BZB J0609-0247 \\
\hline J0612.8+4122 & bll & B3 $0609+413$ & J061251.18+412237.4 & bzb & 0.0 & Massaro+15a & 5BZB J0612+4122 \\
\hline J0618.2-2429 & bcu II & PMN J0618-2426 & J061822.65-242637.7 & bzq & 0.2995 & Crespo+16b & \\
\hline J0644.6+6035 & & & J064459.38+603131.7 & bzb & 0.3582 & Paggi+14 & \\
\hline $\mathrm{J} 0650.7+2503$ & bll & 1ES $0647+250$ & J065046.48+250259.5 & bzb & 0.0 & Massaro+15a & 5BZB J0650+2502 \\
\hline J0653.6+2817 & bcu II & GB6 J0653+2816 & J065340.46+281848.5 & bzg & $0.45 ?$ & Crespo+16a & \\
\hline J0700.0+1709 & bcu II & TXS $0657+172$ & J070001.49+170921.9 & bzq & 1.08 & Crespo+16b & \\
\hline J0700.2+1304 & bcu II & GB6 J0700+1304 & J070014.31+130424.4 & bzb & 0.0 & Crespo+16a & \\
\hline J0708.9+2239 & bcu II & GB6 J0708+2241 & J070858.28+224135.4 & bzb & 0.0 & Massaro+15a & 5BZB J0708+2241 \\
\hline J0720.0-4010 & bcu II & 1RXS J071939.2-401153 & J071939.18-401147.4 & bzb & 0.0 & Crespo+16b & \\
\hline J0721.5-0221 & & & J072113.90-022055.0 & bzb & 0.0 & Crespo+16b & \\
\hline J0723.2-0728 & bcu III & 1RXS J072259.5-073131 & J072259.68-073134.7 & bzb & 0.0 & Crespo $+16 c$ & 5BZB J0722-0731 \\
\hline J0728.0+4828 & bcu II & GB6 J0727+4827 & J072759.84+482720.3 & bzb & 0.0 & Crespo+16a & \\
\hline J0730.5-6606 & bcu II & PMN J0730-6602 & J073049.51-660218.9 & bzb & 0.0 & Crespo+16c & \\
\hline J0746.6-4756 & bcu II & PMN J0746-4755 & J074642.31-475455.0 & bzb & 0.0 & Ricci+15 & \\
\hline J0754.8+4824 & bll & GB1 $0751+485$ & J075445.66+482350.7 & bzb & 0.0 & Massaro+14 & 5BZB J0754+4823 \\
\hline J0813.3+6509 & bcu II & GB6 J0812+6508 & J081240.84+650911.1 & bzb & 0.0 & Massaro+15a & 5BZB J0812+6509 \\
\hline J0814.1-1012 & bll & NVSS J081411-101208 & J081411.69-101210.2 & bzb & 0.0 & Crespo+16a & 5BZB J0814-1012 \\
\hline $\mathrm{J} 0814.7+6428$ & bll & GB6 J0814+6431 & $\mathrm{J} 081439.19+643122.0$ & bzb & 0.0 & Massaro+15a & 5BZB J0814+6431 \\
\hline J0822.9+4041 & fsrq & B3 $0819+408$ & J082257.55+404149.7 & bzq & 0.8655 & Massaro+14 & 5BZQ J0822+4041 \\
\hline J0827.2-0711 & bcu I & PMN J0827-0708 & J082706.16-070845.9 & bzb & 0.0 & Crespo+16c & \\
\hline J0828.8-2420 & bcu III & NVSS J082841-241850 & J082841.74-241851.1 & bzb & 0.0 & Crespo+16b & \\
\hline J0835.4+0930 & bll & GB6 J0835+0936 & J083543.21+093717.9 & bzb & 0.35 ? & Massaro+14 & 5BZB J0835+0937 \\
\hline J0845.1-5458 & bcu II & PMN J0845-5458 & J084502.48-545808.4 & bzb & 0.0 & Ricci+15 & \\
\hline J0849.1+6607 & bll & GB6 J0848+6605 & J084854.60+660609.3 & bzb & 0.0 & Massaro+15a & 5BZB J0848+6606 \\
\hline J0855.4+7142 & & & J085654.85+714623.8 & bzq & 0.542 & Ricci+15 & \\
\hline
\end{tabular}

Column description: (1) 3FGL name reported; (2) 3FGL $\gamma$-ray classification. Empty field implies that the sources is an UGS; (3) 3FGL associated counterpart if present; (4) WISE name of the counterpart observed during our optical spectroscopic campaign; (5) optical classification (6) redshift; question marks indicate uncertain values or redshifts estimated via intervening systems while empty field is used for unknown $z ;$ (7) reference within the papers published with the results of our campaign; (8) the name reported in the Roma-BZCAT.

Notes: Sources pointed twice are reported in different lines within the table. 
Table 3 Summary of the targets observed during our optical spectroscopic campaign for the 3FGL sources. (R.A. 08-16)

\begin{tabular}{|c|c|c|c|c|c|c|c|}
\hline $\begin{array}{l}\text { 3FGL } \\
\text { name }\end{array}$ & $\begin{array}{l}\text { 3FGL } \\
\text { class }\end{array}$ & $\begin{array}{l}\text { 3FGL } \\
\text { counterpart }\end{array}$ & $\begin{array}{l}\text { counterpart } \\
\text { name }\end{array}$ & class & $z$ & reference & $\begin{array}{l}\text { Roma-BZCAT } \\
\text { name }\end{array}$ \\
\hline J0900.0+6754 & & & J090038.69+674223.3 & $\overline{\mathrm{bzzb}}$ & 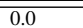 & Massaro+15a & 5BZB J0900+6742 \\
\hline J0904.3+4240 & bcu II & S4 $0900+42$ & J090415.62+423804.5 & bzq & 1.34246 & Crespo+16c & \\
\hline J0917.3-0344 & bcu II & NVSS J091714-034315 & J091714.61-034314.2 & bzg & 0.308 & Crespo+16b & \\
\hline J0921.0-2258 & bcu II & NVSS J092057-225721 & J092057.47-225721.5 & bzb & 0.0 & Crespo+16c & \\
\hline J0924.0+2816 & fsrq & B2 $0920+28$ & J092351.52+281525.1 & bzq & 0.7442 & Massaro+14 & 5BZQ J0923+2815 \\
\hline J0942.1-0756 & bll & PMN J0942-0800 & J094221.46-075953.0 & bzb & 0.0 & Crespo+16a & 5BZB J0942-0759 \\
\hline J0950.1+4554 & bll & RX J0950.2+4553 & J095011.82+455320.0 & bzb & 0.3994 & Massaro+14 & 5BZB J0950+4553 \\
\hline $\mathrm{J} 1003.6+2608$ & bcu I & PKS $1000+26$ & $\mathrm{~J} 100342.22+260512.8$ & bzb & 0.9295 & Crespo+16c & \\
\hline $\mathrm{J} 1007.9+0621$ & bll & MG1 J100800+0621 & $\mathrm{J} 100800.81+062121.2$ & bzb & $0.6495 ?$ & Paggi+14 & $5 \mathrm{BZB} \mathrm{J} 1008+0621$ \\
\hline $\mathrm{J} 1013.5+3440$ & fsrq & OL 318 & $\mathrm{~J} 101336.51+344003.6$ & qso & 0.208 & Crespo+16a & \\
\hline $\mathrm{J} 1016.1+5555$ & bcu II & TXS $1012+560$ & $\mathrm{~J} 101544.43+555100.6$ & bzq & 0.677 & Massaro+15a & 5BZQ J1015+5551 \\
\hline $\mathrm{J} 1018.3+3542$ & fsrq & B2 $1015+35 B$ & $\mathrm{~J} 101810.97+354239.4$ & bzq & 1.228 & Massaro+14 & 5 BZQ J1018+3542 \\
\hline $\mathrm{J} 1023.1+3952$ & fsrq & $4 C+40.25$ & $\mathrm{~J} 102333.50+395312.7$ & bzq & 1.3328 & Massaro+14 & $5 \mathrm{BZQ} \mathrm{J} 1023+3953$ \\
\hline J1038.9-5311 & bcu II & MRC $1036-529$ & J103840.66-531142.9 & bzq & 1.45 & Crespo+16b & \\
\hline $\mathrm{J} 1040.4+0615$ & bcu II & GB6 J1040+0617 & $\mathrm{J} 104031.62+061721.7$ & bzb & 0.0 & Crespo+16c & \\
\hline $\mathrm{J} 1040.8+1342$ & bcu II & 1RXS J104057.7+134216 & $\mathrm{J} 104057.69+134211.7$ & bzb & 0.0 & Crespo+16c & \\
\hline J1042.0-0557 & bcu II & PMN J1042-0558 & $\mathrm{J} 104204.30-055816.5$ & bzg & 0.39 & Crespo+16b & \\
\hline J1049.7+1548 & & & $\mathrm{J} 104939.34+154837.8$ & bzb & 0.3271 & Paggi+14 & 5BZB J1049+1548 \\
\hline $\mathrm{J} 1100.5+4020$ & bll & RX J1100.3+4019 & $\mathrm{J} 110021.05+401928.0$ & bzb & 0.0 & Massaro+14 & $5 \mathrm{BZB} \mathrm{J} 1100+4019$ \\
\hline J1103.3+5239 & & & $\mathrm{J} 110249.86+525012.6$ & bzq & 0.68984 & Massaro+16 & \\
\hline J1103.9-5357 & bll & PKS & $\mathrm{J} 110352.22-535700.7$ & bzb & $0.461 ?$ & Landoni +15 & $103-5357$ \\
\hline $\mathrm{J} 1105.7+4427$ & & & $\mathrm{~J} 110544.29+442830.6$ & bzb & 0.74641 & Massaro+16 & \\
\hline J1123.3-2529 & & & $\mathrm{J} 112325.37-252857.0$ & qso & 0.148 & Ricci+15 & \\
\hline J1125.0-2101 & bcu II & $\mathrm{PMI}$ & $\mathrm{J} 112508.62-210105.9$ & bzb & 0.0 & Crespo+16c & \\
\hline $\mathrm{J} 1129.0+3758$ & & & $\mathrm{~J} 112903.24+375656.7$ & bzb & 0.0 & Massaro+14 & 5 BZB J1129+3756 \\
\hline J1129.0+3758 & & & $\mathrm{J} 112904.78+375844.6$ & bzb & 0.0 & Massaro+16 & \\
\hline J1129.4-4215 & bcu I & SUMSS J113006-421441 & J113007.04-421440.9 & bzb & 0.0 & Crespo+16c & \\
\hline J1141.6-1406 & bcu II & 1RXS J114142.2-140757 & J114141.80-140754.6 & bzb & 0.0 & Ricci+15 & \\
\hline $\mathrm{J} 1200.8+1228$ & bcu II & GB6 J1200+1230 & $\mathrm{J} 120040.03+123103.2$ & bzb & 0.0 & Crespo+16c & \\
\hline J1209.4+4119 & bll & B3 $1206+416$ & $\mathrm{~J} 120922.78+411941.3$ & bzb & 0.0 & Massaro+14 & 5BZB J1209+4119 \\
\hline $\mathrm{J} 1217.8+3007$ & bll & 1ES $1215+303$ & $\mathrm{~J} 121752.08+300700.6$ & bzb & 0.0 & Ricci+15 & $5 \mathrm{BZB} \mathrm{J} 1217+3007$ \\
\hline $\mathrm{J} 1221.5-0632$ & & & $\mathrm{~J} 122127.20-062847.8$ & qso & 0.44 & Crespo+16b & \\
\hline J1222.4+0414 & fsrq & $4 \mathrm{C}+04.42$ & $\mathrm{~J} 122222.54+041315.7$ & bzq & 0.9642 & Massaro+14 & 5 BZQ J1222+0413 \\
\hline $\mathrm{J} 1222.7+7952$ & & & $\mathrm{~J} 122358.06+795328.2$ & bzb & 0.0 & Massaro+15a & $5 \mathrm{BZB} \mathrm{J} 1223+7953$ \\
\hline $\mathrm{J} 1230.3+2519$ & bll & ON 246 & $\mathrm{~J} 123014.08+251807.1$ & bzb & 0.0 & Massaro+15a & $5 \mathrm{BZB} \mathrm{J} 1230+2518$ \\
\hline J1238.2-1958 & bcu II & PMN J1238-1959 & J123824.39-195913.8 & bzb & 0.0 & Ricci+15 & 5BZU J1238-1959 \\
\hline $\mathrm{J} 1243.1+3627$ & bll & Ton 116 & $\mathrm{~J} 124312.73+362743.9$ & bzb & 0.0 & Massaro+14 & 5 BZB J1243+3627 \\
\hline J1256.3-1146 & bcu I & PMN J1256-1146 & $\mathrm{J} 125615.95-114637.3$ & bzg & 0.0579 & Crespo+16c & 5BZG J1256-1146 \\
\hline J1259.8-3749 & bcu II & NVSS J125949-374856 & J125949.80-374858.1 & bzb & 0.0 & Ricci+15 & \\
\hline $\mathrm{J} 1301.5+3333$ & & & $\mathrm{~J} 130129.16+333700.2$ & bzq & 1.0084 & Massaro+14 & $5 \mathrm{BZQ}$ J1301+3337 \\
\hline $\mathrm{J} 1301.5+3333$ & & & $\mathrm{~J} 130129.16+333700.2$ & bzq & 1.00826 & Massaro+16 & $5 \mathrm{BZQ} \mathrm{J} 1301+3337$ \\
\hline $\mathrm{J} 1311.0+0036$ & bll & RX J1311.1+0035 & $\mathrm{J} 131106.47+003510.0$ & bzb & 0.0 & Massaro+14 & $5 \mathrm{BZB} \mathrm{J} 1311+0035$ \\
\hline $\mathrm{J} 1314.8+2349$ & bll & TXS $1312+240$ & $\mathrm{~J} 131443.80+234826.7$ & bzb & 0.0 & Massaro+14 & 5BZB J1314+2348 \\
\hline $\mathrm{J} 1314.8+2349$ & bll & TXS $1312+240$ & $\mathrm{~J} 131443.80+234826.7$ & bzb & 0.0 & Paggi+14 & 5BZB J1314+2348 \\
\hline $\mathrm{J} 1315.4+1130$ & bcu II & 1RXS J131531.9+113327 & $\mathrm{J} 131532.62+113331.7$ & bzb & 0.73 & Crespo+16c & \\
\hline J1322.3+0839 & bcu II & NVSS J132210+084231 & $\mathrm{J} 132210.17+084232.9$ & bzb & 0.0 & Crespo+16a & \\
\hline J1322.3+0839 & bcu II & NVSS J132210+084231 & $\mathrm{J} 132210.17+084232.9$ & bzq & 0.32549 & Crespo+16c & \\
\hline J1328.5-4728 & bcu II & 1WGA J1328.6-4727 & $\mathrm{J} 132840.61-472749.2$ & bzb & 0.0 & Ricci+15 & \\
\hline $\mathrm{J} 1330.6+7002$ & bll & NVSS J133025+700141 & $\mathrm{J} 133025.82+700138.7$ & bzb & 0.0 & Massaro+15a & $5 \mathrm{BZB} \mathrm{J} 1330+7001$ \\
\hline $\mathrm{J} 1330.4+5641$ & & & $\mathrm{~J} 133040.67+565520.1$ & bzb & 0.0 & Massaro+16 & $5 \mathrm{BZB} \mathrm{J} 1330+5655$ \\
\hline $\mathrm{J} 1331.8+4718$ & fsrq & B3 $1330+476$ & $\mathrm{~J} 133245.24+472222.6$ & bzq & 0.6687 & Massaro+14 & 5 BZQ J1332+4722 \\
\hline J1340.6-0408 & bcu II & NVSS J134042-041006 & $\mathrm{J} 134042.02-041006.8$ & bzb & 0.0 & Ricci+15 & \\
\hline J1340.6-0408 & bcu II & NVSS J134042-041006 & $\mathrm{J} 134042.02-041006.8$ & bzb & 0.0 & Crespo+16c & \\
\hline $\mathrm{J} 1342.7+0945$ & bcu II & NVSS J134240+094752 & $\mathrm{J} 134240.02+094752.4$ & qso & 0.28279 & Crespo+16c & \\
\hline J1346.9-2958 & bcu II & NVSS J134706-295840 & J134706.88-295842.4 & bzb & 0.0 & Ricci+15 & \\
\hline J1351.4+1115 & bll & RX J1351.3+1115 & $\mathrm{J} 135120.84+111453.0$ & bzb & 0.0 & Massaro+14 & $5 \mathrm{BZB} \mathrm{J} 1351+1114$ \\
\hline J1359.9-3746 & bll & PMN J1359-3746 & J135949.71-374600.7 & bzb & 0.0 & Ricci+15 & 5BZB J1359-3746 \\
\hline J1406.0-2508 & bcu II & NVSS J140609-250 & $\mathrm{J} 140609.60-250809.2$ & bzb & 0.0 & Ricci+15 & \\
\hline $\mathrm{J} 1410.4+2821$ & bll & RX J1410.4+2821 & $\mathrm{J} 141029.56+282055.6$ & bzb & 0.0 & Massaro+14 & $5 B Z B \mathrm{~J} 1410+2820$ \\
\hline $\mathrm{J} 1411.1+3717$ & & & $\mathrm{~J} 141130.51+372246.4$ & bzb & 0.0 & Massaro+16 & \\
\hline $\mathrm{J} 1412.0+5249$ & bcu I & SBS 1 & $\mathrm{~J} 141149.44+524900.2$ & bzg & 0.07649 & Crespo+16c & \\
\hline $\mathrm{J} 1434.6+6640$ & bcu II & 1RXS J143442.0+664031 & $\mathrm{J} 143441.46+664026.5$ & bzb & 0.0 & Crespo+16a & \\
\hline $\mathrm{J} 1442.0+4348$ & bll & SDSS J144207.15+434836.6 & $\mathrm{J} 144207.15+434836.7$ & bzb & 0.0 & Massaro+14 & 5 BZB J1442+4348 \\
\hline J1444.0-3907 & bll & PKS $1440-389$ & $\mathrm{~J} 144357.20-390840.0$ & bzb & 0.0 & Landoni +15 & 5BZB J1443-3908 \\
\hline J1511.8-0513 & bcu III & NVSS J151148-051345 & J151148.56-051346.9 & bzb & 0.0 & Crespo+16a & \\
\hline $\mathrm{J} 1521.8+4340$ & fsrq & B3 $1520+437$ & $\mathrm{~J} 152149.61+433639.2$ & bzq & 2.1677 & Massaro+14 & $5 \mathrm{BZQ}$ J1521+4336 \\
\hline J1548.4+1455 & & & $\mathrm{J} 154824.38+145702.8$ & bzg & 0.23 & Crespo+16b & \\
\hline $\mathrm{J} 1549.0+6309$ & bcu II & SDSS J154958.45+631021.2 & $\mathrm{J} 154828.42+631051.1$ & bzg & 0.269 & Massaro+15b & $5 \mathrm{BZG} \mathrm{J1548+6310}$ \\
\hline $\mathrm{J} 1549.5+1709$ & bcu II & MG1 J154930+1708 & $\mathrm{J} 154929.28+170828.1$ & bzb & 0.0 & Crespo+16c & \\
\hline $\mathrm{J} 1553.5-3118$ & bll & 1RXS J155333.4-311841 & $\mathrm{J} 155333.56-311830.9$ & bzb & 0.0 & Ricci+15 & 5BZB J1553-3118 \\
\hline $\mathrm{J} 1611.9+1404$ & & & $\mathrm{~J} 161118.10+140328.7$ & qso & 0.5855 & Massaro+14 & \\
\hline $\mathrm{J} 1626.4-7640$ & bcu II & PKS 1619-765 & $\mathrm{J} 162638.15-763855.5$ & bzb & 0.1050 & Ricci+15 & 5BZU J1626-7638 \\
\hline $\mathrm{J} 1627.8+3217$ & & & $\mathrm{~J} 162800.39+322414.0$ & qso & 0.9051 & Massaro+14 & \\
\hline $\mathrm{J} 1636.7+2624$ & bcu II & NVSS J163651+262657 & $\mathrm{J} 163651.46+262656.7$ & bzb & 0.0 & Crespo+16c & \\
\hline $\mathrm{J} 1647.4+4950$ & bcu I & SBS $1646+499$ & $\mathrm{~J} 164734.91+495000.5$ & bzq & 0.049 & Crespo+16a & $5 \mathrm{BZU}$ J1647+4950 \\
\hline $\mathrm{J} 1649.4+5238$ & bll & $87 \mathrm{~GB} 164812.2+524023$ & $\mathrm{~J} 164924.98+523515.0$ & bzb & 0.0 & Ricci+15 & 5 BZB J1649+5235 \\
\hline J1656.8-2010 & bcu II & 1RXS J165655.0-201049 & $\mathrm{J} 165655.14-201056.2$ & bzb & 0.0 & Crespo+16c & \\
\hline
\end{tabular}

Column description: (1) 3FGL name reported; (2) 3FGL $\gamma$-ray classification. Empty field implies that the sources is an UGS; (3) 3FGL associated counterpart if present; (4) WISE name of the counterpar observed during our optical spectroscopic campaign; (5) optical classification (6) redshift; question marks indicate uncertain values or redshifts estimated via intervening systems while empty field is used for unknown $z$; (7) reference within the papers published with the results of our campaign; (8) the name reported in the Roma-BZCAT. 
Table 4 Summary of the targets observed during our optical spectroscopic campaign for the 3FGL sources. (R.A. 16-24)

\begin{tabular}{|c|c|c|c|c|c|c|c|}
\hline $\begin{array}{l}\text { 3FGL } \\
\text { name }\end{array}$ & $\begin{array}{l}\text { 3FGL } \\
\text { class }\end{array}$ & $\begin{array}{l}\text { 3FGL } \\
\text { counterpart }\end{array}$ & $\begin{array}{l}\text { counterpart } \\
\text { name }\end{array}$ & class & $z$ & reference & $\begin{array}{l}\text { Roma-BZCAT } \\
\text { name }\end{array}$ \\
\hline$\overline{J J 1704.1+1234}$ & & & J170409.58+123421.7 & bzb & 0.45 & 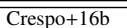 & \\
\hline $\mathrm{J} 1719.2+1744$ & bll & PKS $1717+177$ & $\mathrm{~J} 171913.05+174506.5$ & bzb & 0.0 & Massaro+15b & 5BZB J1719+1745 \\
\hline $\mathrm{J} 1725.3+5853$ & bll & 7C $1724+5854$ & $\mathrm{~J} 172535.02+585140.0$ & bzb & 0.0 & Paggi+14 & 5BZB J1725+5851 \\
\hline J1730.6-0357 & & & J173052.85-035247.2 & bzb & $0.776 ?$ & Ricci+15 & \\
\hline $\mathrm{J} 1731.9+5428$ & & & $\mathrm{~J} 173340.31+542636.7$ & bzb & 0.0 & Massaro+16 & \\
\hline $\mathrm{J} 1736.0+2033$ & bcu II & NVSS J173605+203301 & $\mathrm{J} 173605.25+203301.1$ & bzb & 0.0 & Crespo+16a & 5BZB J1736+2033 \\
\hline $\mathrm{J} 1801.5+4403$ & fsrq & S4 $1800+44$ & $\mathrm{~J} 180132.32+440421.7$ & bzq & 0.663 & Massaro+15b & 5BZQ J1801+4404 \\
\hline $\mathrm{J} 1809.7+2909$ & bll & MG2 J180948+2910 & $\mathrm{J} 180945.39+291019.8$ & bzb & 0.0 & Ricci+15 & 5BZB J1809+2910 \\
\hline $\mathrm{J} 1819.1+2134$ & bcu II & MG2 J181902+2132 & $\mathrm{J} 181905.22+213233.8$ & bzb & 0.0 & Crespo+16b & \\
\hline $\mathrm{J} 1835.4+1349$ & bcu III & TXS $1833+137$ & $\mathrm{~J} 183535.34+134848.8$ & bzb & 0.0 & Ricci+15 & \\
\hline $\mathrm{J} 1836.3+3137$ & bll & RX J1836.2+3136 & $\mathrm{J} 183621.23+313626.8$ & bzb & 0.0 & Massaro+15a & 5BZB J1836+3136 \\
\hline $\mathrm{J} 1844.3+1547$ & bcu II & NVSS J184425+154646 & $\mathrm{J} 184425.36+154645.8$ & bzb & 0.0 & Massaro+15b & \\
\hline $\mathrm{J} 1844.1+5709$ & bll & TXS $1843+571$ & J184450.96+570938.6 & bzb & 0.0 & Massaro+15a & 5BZB J1844+5709 \\
\hline $\mathrm{J} 1849.5+2751$ & bll & MG2 J184929+2748 & $\mathrm{J} 184931.74+274800.8$ & bzb & 0.0 & Ricci+15 & 5BZB J1849+2748 \\
\hline $\mathrm{J} 1903.2+5541$ & bll & TXS $1902+556$ & J190311.60+554038.5 & bzb & 0.0 & Massaro+15a & 5BZB J1903+5540 \\
\hline J1913.9+4441 & bcu II & 1RXS J191401.9+443849 & $\mathrm{J} 191401.88+443832.2$ & bzb & 0.0 & Crespo+16a & \\
\hline $\mathrm{J} 1942.7+1033$ & bcu II & 1RXS J194246.3+103339 & $\mathrm{J} 194247.48+103327.0$ & bzb & 0.0 & Massaro+15b & 5BZB J1942+1033 \\
\hline J1955.0-1605 & bcu II & 1RXS J195500.6-160328 & J195500.65-160338.4 & bzb & 0.0 & Crespo+16c & \\
\hline J1959.8-4725 & bcu II & SUMSS J195945-472519 & J195945.66-472519.3 & bzb & 0.519 ? & Ricci+15 & \\
\hline $\mathrm{J} 2004.8+7003$ & bll & 1 RXS J200504.0+700445 & $\mathrm{J} 200505.97+700439.5$ & bzb & 0.0 & Massaro+15a & 5BZB J2005+7004 \\
\hline $\mathrm{J} 2014.5+0648$ & bcu II & NVSS J201431+064849 & J201431.08+064852.5 & bzg & 0.341 & Massaro $+15 b$ & 5BZB J2014+0648 \\
\hline J2015.3-1431 & & & J201525.02-143203.9 & bzb & 0.0 & Crespo+16b & \\
\hline $\mathrm{J} 2021.9+0630$ & bll & 87GB $201926.8+061922$ & J202155.45+062913.6 & bzb & 0.0 & Crespo+16a & \\
\hline J2031.0+1937 & bcu II & RX J2030.8+1935 & J203057.12+193612.9 & bzb & $0.668 ?$ & Massaro+15b & \\
\hline J2036.4+6551 & bll & $87 \mathrm{~GB} 203539.4+654245$ & J203620.14+655314.5 & bzb & 0.0 & Crespo+16a & 5BZB J2036+6553 \\
\hline J2036.6-3325 & bcu II & 1RXS J203650.9-332817 & J203649.49-332830.7 & bzb & 0.23 & Crespo+16b & \\
\hline $\mathrm{J} 2039.5+5217$ & bll & 1ES $2037+521$ & $\mathrm{~J} 203923.51+521950.1$ & bzb & 0.0 & Ricci+15 & 5BZB J2039+5219 \\
\hline J2104.2-0211 & bcu II & NVSS J210421-021239 & J210421.92-021239.0 & bzb & 0.0 & Crespo+16c & \\
\hline $\mathrm{J} 2108.0+3654$ & bcu II & TXS $2106+367$ & $\mathrm{~J} 210805.46+365526.5$ & bzb & 0.0 & Massaro+15a & \\
\hline J2109.1-6638 & bcu II & PKS 2104-668 & J210851.80-663722.7 & bzb & 0.0 & Crespo+16c & 5BZB J2108-6637 \\
\hline J2118.0-3241 & bcu I & NVSS J211754-324326 & J211754.91-324328.2 & bzb & 0.0 & Crespo+16c & \\
\hline $\mathrm{J} 2127.7+3612$ & bll & B2 $2125+35$ & $\mathrm{~J} 212743.03+361305.7$ & $\mathrm{bzb}$ & 0.0 & Massaro+15a & 5BZB J2127+3613 \\
\hline $\mathrm{J} 2133.3+2533$ & bcu II & $87 \mathrm{~GB} 213100.1+251534$ & J213314.36+252859.0 & bzg & 0.294 & Massaro+15b & $5 B Z G ~ J 2133+2528$ \\
\hline J2134.5-2131 & & & J213430.18-213032.8 & $\mathrm{bzb}$ & 0.0 & Crespo+16b & \\
\hline $\mathrm{J} 2145.5+1007$ & & & $\mathrm{~J} 214530.19+100605.5$ & bzb & 0.0 & Massaro+16 & \\
\hline $\mathrm{J} 2156.0+1818$ & bcu II & RX J2156.0+1818 & J215601.64+181837.1 & bzb & 0.0 & Crespo+16a & \\
\hline J $2223.3+0103$ & & & J222329.57+010226.7 & bzb & $0.29 ?$ & Massaro+14 & 5BZB J2223+0102 \\
\hline $\mathrm{J} 2223.3+0103$ & & & J222329.57+010226.7 & bzb & 0.0 & Massaro+16 & 5BZB J2223+0102 \\
\hline J2232.9-2021 & bcu II & 1RXS J223249.5-202232 & J223248.80-202226.2 & bzb & 0.0 & Crespo+16c & \\
\hline $\mathrm{J} 2247.8+4413$ & bll & NVSS J224753+441317 & $\mathrm{J} 224753.22+441315.5$ & bzb & 0.0 & Massaro+15a & 5BZB J2247+4413 \\
\hline $\mathrm{J} 2251.9+4031$ & bll & MG4 J225201+4030 & $\mathrm{J} 225159.77+403058.0$ & bzb & 0.0 & Massaro+15a & 5 BZB J2251+4030 \\
\hline $\mathrm{J} 2255.1+2411$ & bll & MG3 J225517+2409 & J225515.37+241011.2 & bzb & 0.0 & Massaro+15a & 5 BZB J $2255+2410$ \\
\hline J2258.2-3645 & & & J225815.00-364434.3 & bzb & 0.0 & Landoni+15 & 5BZB J2258-3644 \\
\hline $\mathrm{J} 2300.3+3136$ & bll & NVSS J230022+313703 & J230022.84+313704.4 & bzb & 0.0 & Massaro+15b & $5 B Z B$ J2300+3137 \\
\hline $\mathrm{J} 2311.0+0204$ & bll & NVSS J231101+020504 & $\mathrm{J} 231101.29+020505.3$ & bzb & 0.0 & Massaro+15a & 5BZB J $2311+0205$ \\
\hline J2317.3-4534 & bcu II & 1RXS J231733.0-453348 & J231731.98-453359.6 & bzb & 0.0 & Crespo $+16 c$ & \\
\hline $\mathrm{J} 2323.9+4211$ & bll & 1ES $2321+419$ & J232352.07+421058.5 & bzb & 0.0 & Crespo+16a & $5 B Z B$ J $2323+4210$ \\
\hline $\mathrm{J} 2323.9+4211$ & bll & 1ES $2321+419$ & $\mathrm{~J} 232352.07+421058.5$ & bzb & 0.0 & Massaro+15a & 5BZB J $2323+4210$ \\
\hline $\mathrm{J} 2324.7+0801$ & bll & PMN J2324+0801 & J232445.32+080206.1 & bzb & 0.0 & Massaro+15a & 5BZB J2324+0802 \\
\hline $\mathrm{J} 2325.6+1650$ & bll & NVSS J232538+164641 & $\mathrm{J} 232538.11+164642.7$ & bzb & 0.0 & Massaro+15a & 5BZB J2325+1646 \\
\hline J2328.4-4034 & bcu II & PKS 2325-408 & J232819.26-403509.8 & bzq & 1.972 & Crespo+16c & 5BZQ J2328-4035 \\
\hline $\mathrm{J} 2339.0+2122$ & bll & RX J2338.8+2124 & J233856.38+212441.3 & bzb & 0.0 & Massaro+15a & $5 B Z B \mathrm{~J} 2338+2124$ \\
\hline $\mathrm{J} 2340.7+8016$ & bll & 1RXS J234051.4+801513 & $\mathrm{J} 234054.23+801516.0$ & bzb & 0.0 & Massaro+15b & 5BZB J $2340+8015$ \\
\hline $\mathrm{J} 2346.7+0705$ & bcu II & TXS $2344+068$ & $\mathrm{~J} 234639.93+070506.8$ & bzb & 0.17 & Crespo $+16 c$ & \\
\hline $\mathrm{J} 2352.0+1752$ & bll & CLASS J2352+1749 & J235205.84+174913.7 & bzb & 0.0 & Massaro+15a & 5BZB J2352+1749 \\
\hline $\mathrm{J} 2356.0+4037$ & bll & NVSS J235612+403648 & $\mathrm{J} 235612.70+403646.8$ & bzg & 0.131 & Massaro+15a & 5 BZG J2356+4036 \\
\hline
\end{tabular}

Column description: (1) 3FGL name reported; (2) 3FGL $\gamma$-ray classification. Empty field implies that the sources is an UGS; (3) 3FGL associated counterpart if present; (4) WISE name of the counterpar observed during our optical spectroscopic campaign; (5) optical classification (6) redshift; question marks indicate uncertain values or redshifts estimated via intervening systems while empty field is used fo unknown $z ;$ (7) reference within the papers published with the results of our campaign; (8) the name reported in the Roma-BZCAT.

Notes: Sources pointed twice are reported in different lines within the table.

Table 5 Summary of the targets observed during our optical spectroscopic campaign for the 1FGL, 2FGL and 1FHL sources only.

\begin{tabular}{|c|c|c|c|c|c|c|c|}
\hline $\begin{array}{l}\text { Fermi } \\
\text { name }\end{array}$ & $\begin{array}{l}\text { Fermi } \\
\text { class }\end{array}$ & $\begin{array}{l}\text { Fermi } \\
\text { counterpart }\end{array}$ & $\begin{array}{l}\text { counterpart } \\
\text { name }\end{array}$ & class & $z$ & reference & $\begin{array}{l}\text { Roma-BZCAT } \\
\text { name }\end{array}$ \\
\hline 1FHL J0030.1-1647 & & & J003020.44-164713.1 & $\overline{\mathrm{bzb}}$ & 0.237 & Crespo+16b & \\
\hline 1FHL J0044.0-1111 & & & J004348.66-111607.2 & bzb & 0.264 & Crespo+16b & \\
\hline 2FGL J0332.5-1118 & agu & NVSS J033223-111951 & J033223.25-111950.6 & bzq & 0.2074 & Crespo+16b & 5BZU J0332-1119 \\
\hline 1FGL J0411.6+5459 & & & $\mathrm{J} 041203.78+545747.2$ & bzb & 0.0 & Crespo+16b & \\
\hline 2FGL J0819.6-0803 & bzb & RX J0819.2-0756 & J081917.58-075626.0 & bzb & 0.0 & Crespo+16b & 5BZB J0819-0756 \\
\hline 1FGL J1422.7+3743 & bzb & CLASS J1423+3737 & $\mathrm{J} 142304.61+373730.5$ & bzb & 0.0 & Massaro+14 & 5BZB J1423+3737 \\
\hline 2FGL J1624.4+1123 & agu & MG1 J162441+1111 & $\mathrm{J} 162444.79+110959.3$ & bzq & 2.1 & Crespo+16b & \\
\hline 2FGL J1745.6+0203 & & & $\mathrm{J} 174507.82+015442.4$ & qso & 0.078 & Ricci+15 & \\
\hline 2FGL J1803.6+2523c & agu & TXS $1801+253$ & 2MASS J18031240+2521185 & bzq & 0.77 & Crespo+16b & \\
\hline 2FGL J1848.6+3241 & agu & B2 $1846+32 B$ & $\mathrm{~J} 184834.37+324400.0$ & bzq & 0.981 & Massaro+15b & \\
\hline 2FGL J2110.3+3822 & & & $\mathrm{J} 211020.19+381659.2$ & qso & 0.46 & Massaro+15a & \\
\hline
\end{tabular}

Column description: (1) Fermi name reported in the 1FGL, 2FGL or 1FHL; (2) Fermi $\gamma$-ray classification; (3) Fermi associated counterpart if present; (4) WISE or 2MASS name of the counterpart observed during our optical spectroscopic campaign; (5) optical classification (6) redshift; question marks indicate uncertain values or redshifts estimated via intervening systems while empty field is used for unknown $z ;$ (7) reference within the papers published with the results of our campaign; (8) the name reported in the Roma-BZCAT. 


\section{References}

Abdo, A. A., Ackermann, M., Ajello, M. et al. 2010a ApJS, 188, 405

Abdo, A. A., Ackermann, M., Ajello, M. et al. 2010b ApJ, 715, 429

Acero, F., Donato, D., Ojha, R. et al. 2013 ApJ, 779, 133

Acero, F., Ackermann, M., Ajello, M. et al. 2015 ApJS, 218, 23

Ackermann, M., Ajello, M., Allafort, A. et al. 2011a ApJ, 743, 171

Ackermann, M., Ajello, M., Allafort, A. et al. 2012a ApJ, 755, 164 Ackermann, M. et al. 2012b Sci, 338, 1190

Ackermann, M., Ajello, M., Allafort, A. et al. 2013 ApJS, 209, 34

Ackermann, M., Ajello, M., Atwood, W. B. et al. 2015 ApJ, 810, 14

Ackermann, M., Ajello, M., Atwood, W. B. et al. 2016 ApJS, 222, 5

Ajello, M., Shaw, M. S., Romani, R. W. et al. 2012 ApJ, 751, 108

Ajello, M. et al. 2014 ApJ, 780, 73

Ajello, M.; Gasparrini, D.; Sánchez-Conde, M. 2015 ApJ, 800L, 27

Alam, S., Albareti, F. D., Allende Prieto, C. et al. 2015 ApJS, 219, 12

Àlvárez Crespo, N., Masetti, N., Landoni, M. et al. 2016 AJ, 151, 32

Àlvárez Crespo, N., Massaro, F., Milisavljevic, D. et al. 2016 AJ, 151,95

Álvarez-Crespo, N., Massaro, F., D’Abrusco, R. et al. 2016c ApSS 361,316

Arsioli, B. et al. 2015 A\&A, 579A, 34

Atwood, W. B., Abdo, A. A., Ackermann, M. et al. 2009 ApJ, 697, 1071

Berlin, A. \& Hooper D. 2014 PhRvD, 89a6014;

Bernieri, E. et al. 2013 A\&A, 551L, 5

Bonnarel, F., Fernique, P., Bienaymé, O. et al. 2000 A\&AS, 143, 33

Becker, R. H., White, R. L., Helfand, D. J. 1995 ApJ, 450, 559

Casandjian, J.-M. \& Grenier, I. A. 2008 A\&A, 489, 849

Campana, R. et al. 2015 Ap\&SS, 360, 65

Campana, R.; Massaro, E.; Bernieri, E. 2016a Ap\&SS 361, 183

Campana, R.; Massaro, E.; Bernieri, E. 2016b Ap\&SS 361, 185

Cheung, C. C., Donato, D., Gehrels, N. et al. 2012 ApJ, 756, 33

Chiaro, G., Salvetti, D., La Mura, G., Giroletti, M., Thompson, D.

J., Bastieri, D. 2016 MNRAS, 462, 3180

Cowperthwaite, Philip S. et al. 2013 AJ, 146, 110

D’Abrusco, R., Massaro, F., Ajello, M. et al. 2012 ApJ, 748, 68

D'Abrusco, R., Massaro, F., Paggi, A. et al. 2013 ApJS, 206, 12

D'Abrusco, R., Massaro, F., Paggi, A. et al. 2014 ApJS, 215, 14

D'Abrusco, R. et al. 2016 ApJS in prep.

Di Mauro, M., Calore, F., Donato, F., Ajello, M., Latronico, L. 2014 ApJ, 780, 161

Di Mauro, M. \& Donato, F. 2015 PhRvD, 9113001D

The Fermi LAT Collaboration 2015 JCAP, 09, 008

Giommi, P. et al. 2012 A\&A, 541A.160

Giroletti, M., Massaro, F., D’Abrusco, R. et al. 2016 A\&A, 588A, 141

Hartman, R. C., Bertsch, D. L., Bloom, S. D. et al. 1999 ApJS, 123, 79

Hovatta, T., Lister, M. L., Aller, M. F. et al. 2012 AJ, 144, 105

Hovatta, T., Aller, M. F., Aller, H. D. et al. 2014 AJ, 147, 143

Kataoka, J., Yatsu, Y., Kawai, N. 2012 ApJ, 757, 176

Kovalev, Y. Y. 2009 ApJ, 707L, 56

Inoue, Y. 2011 ApJ, 733, 66
Landoni, M., Massaro, F. et al. 2015 AJ, 149, 63

López-Caniego, M. et al. 2013 MNRAS, 430, 1566

Maeda, K., Kataoka, J., Nakamori, T. et al. 2011 ApJ, 729, 103

Maselli, A., Massaro, F., Cusumano, G. et al. 2013 ApJS, 206, 17

Maselli, A., Massaro, F., D’Abrusco, R. et al. 2015 Ap\&SS, 357, 141

Massaro, E., Giommi, P., Leto, C. et al. 2009 A\&A, 495, 691

Massaro, F., D’ Abrusco, R., Ajello, M. et al. 2011a ApJ, 740L, 48

Massaro, F. \& Ajello, M. 2011b ApJ, 729L, 12

Massaro, F., D’Abrusco, R., Tosti, G. et al. 2012a ApJ, 752, 61

Massaro, E., Nesci, R. \& Piranomonte S. 2012 MNRAS, 422, 2322

Massaro, F., D'Abrusco, R., Tosti, G. et al. 2012b ApJ, 750, 138

Massaro, F., D'Abrusco, R., Giroletti, M. et al. 2013a ApJS, 207, 4

Massaro, F., D'Abrusco, R., Paggi, A. et al. 2013b ApJS, 206, 13

Massaro, F., Paggi, A., Errando, M. et al. 2013c ApJS, 207, 16;

Massaro, F., Masetti, N., D’ Abrusco, R. et al. 2014 AJ, 148, 66

Massaro, F., D'Abrusco, R., Landoni, M. et al. 2015a ApJS, 217, 2

Massaro, E., Maselli, A., Leto, C. et al. 2015b Ap\&SS, 357, 75

Massaro, F., Landoni, M., D’Abrusco, R. et al.. 2015c, A\&A, 575, 124

Massaro, F., Thompson, D. J., Ferrara, E. C. 2016 A\&AR, 24, 2

Massaro, F. \& D?Abrusco, R. 2016 ApJ, 827, 67

Mirabal, N. 2009 ApJ, 701, 129

Nolan, P. L., Abdo, A. A., Ackermann, M. et al. 2012 ApJS, 199, 31

Nori, M., Giroletti, M., Massaro, F. et al. 2014 ApJS 212, 3

Paggi, A., Massaro, F., D’Abrusco, R. et al. 2013 ApJS, 209, 9

Paggi, A., Milisavljevic, D., Masetti, N. et al. 2014 AJ, 147, 112

Petrov, L., Mahony, E. K., Edwards, P. G. et al. 2013 MNRAS, 432, 1294

Ricci F., Massaro F., Landoni M. et al. 2015 AJ, 149, 160

Schinzel, F. K., Petrov, L., Taylor, G. B. 2015 ApJS, 217, 4

Shaw, M. S., Romani, R. W.; Cotter, G. et al. 2013 ApJ, 764, 135

Shaw, M. S., Filippenko, A. V., Romani, R. W. et al. 2013 AJ, 146, 127

Stickel, M., Padovani, P., Urry, C. M. et al. 1991 ApJ, 374, 431

Stroh, M. C. \& Falcone, A. D. 2013, ApJS, 207, 28

Takahashi, Y., Kataoka, J., Nakamori, T. et al. 2012 ApJ, 747, 64

Takeuchi, Y., Kataoka, J., Maeda, K. et al. 2013 ApJS, 208, 25

Taylor, M. B. 2005, ASP Conf. Ser., 347, 29

Thompson, D. J., Bertsch, D. L., Fichtel, C. E. et al. 1993 ApJS, 86,629

Thompson, D. J. 2008 RPPh, 71k6901

Urry, C. M., \& Padovani, P. 1995, PASP, 107, 803

White, R. L., Becker, R. H. Helfand, D. J., Gregg, M. D. et al. 1997 ApJ, 475, 479

Wright, E. L., et al. 2010 AJ, 140, 1868

Zechlin, H. \& Horns, D. 2012 JCAP, 11, 050.

This manuscript was prepared with the AAS LATEX macros v5.2. 